\title{
Role of Osteoclast Regulation in Arthritis - A Review
}

\author{
Asif Iqbal
}

Department of Pharmacy, North South University, Dhaka, Bangladesh

\section{ABSTRACT}

Osteoclasts are multinucleated bone degrading cells that differentiate from monocyte/macrocphage precursors of the hematopoietic system. From the bone marrow, precursors of osteoclasts move towards the systemic circulation through the attraction towards chemokines in the blood and are later taken up by various resorption sites where they undergo differentiation into osteoclasts. Macrophage colony stimulating factor (M-CSF) and receptor activator of nuclear factor-жB ligand (RANKL) play a pivotal role in the differentiation and activation of osteoclasts. M-CSF induces the expression of RANK on myeloid progenitors and RANKL activates its receptor to initiate osteoclast differentiation. RANKL also stimulates bone resorption in mature osteoclasts. Rheumatoid arthritis is an autoimmune disease that leads to severe bone destruction mediated by the abnormal activation of osteoclasts. Synovial tissues in rheumatoid arthritis produce inflammatory cytokines that act on osteoclast precursor cells, thereby differentiating them into osteoclasts by cooperating with RANKL. Bone resorption proceeds uncontrollably through the abnormal activity of osteoclasts, leading to bone and cartilage destruction in the joints. The involvement of osteoclasts in the pathogenesis of rheumatoid arthritis has been further confirmed through animal model studies.

KEYWORDS: Osteoclast; M-CSF; RANK; RANKL; Rheumatoid arthritis

\section{INTRODUCTION}

The term "Arthritis" comes from the Greek words arthro- meaning joint and-itis meaning inflammation and is literally used to describe inflammation in the joints, or any disorder that affects the joints [1,2]. Joint inflammation could be a sign or symptom rather than the disease itself and these disorders have been classified under a broader category of rheumatic diseases ranging over 100 types. Cardinal signs of these diseases include inflammation (signs of Inflammation: rubor or redness, calor or heat, dolor or pain and tumor or swelling), loss of function of one or more connecting or supporting structures of the body such as joints, tendons, ligaments, bones and muscles. Pain is a common feature of all these diseases but vary in severity. Tenderness, malaise, fatigue, morning stiffness, weight

\section{Vol No: 05, Issue: 01}

Received Date: September 07, 2021

Published Date: September 30, 2021

\section{*Corresponding Author}

\section{Asif Iqbal}

Department of Pharmacy, North South University, Dhaka, Bangladesh

E-mail: asif.i.pharmacy@gmail.com

Citation: Asif Iqbal. (2021). Role of Osteoclast Regulation in Arthritis - A Review. Mathews J Pharm Sci. 5(1):06.

Copyright: Asif Iqbal. (C2021. This is an openaccess article distributed under the terms of the Creative Commons Attribution License, which permits unrestricted use, distribution, and reproduction in any medium, provided the original author and source are credited. 
loss, fever, anemia, poor sleep, muscle weakness and loss of flexibility are observed in some forms [1]. They may, in some cases, affect internal organs including the heart, eyes, kidneys, lungs and skin. Two of the most common forms of arthritis include osteoarthritis which affects roughly 3.8 percent of people, and rheumatoid arthritis which accounts for only 0.24 percent of reported cases. Although osteoarthritis is age dependent, rheumatoid arthritis is mainly an autoimmune disorder. Other related diseases such as gout, fibromyalgia, lupus and septic arthritis are also included in the category of rheumatic diseases [2].

Arthritis is very common but not well understood. Arthritis and other related rheumatic disorders are among the most prevalent chronic conditions around the globe, causing morbidity, disability and posing a major economic and health burden to the society at large. People of all ages, race and gender can have arthritis. In the United States nearly 12.1 percent of the population aged 25 and above are affected by osteoarthritis, accounting for an estimate of around 20 billion dollars spent per year on treatment. In UK, prevalence is around 13 percent [3]. According to a survey by Ahmed $\mathrm{M}$, et al. rheumatic diseases are more common in the rural areas of Bangladesh than in the big cities, affecting at least 1.6 percent of the total population [4]. Another study by Ahmed S, et al. reported that about one third of the adult population in Bangladesh suffer from musculoskeletal pain at any point in their lives. Severe arthritis may result in chronic pain, inability to perform daily tasks and making it difficult to walk or climb stairs thereby, decreasing the quality of life. It can also cause permanent joint damage. Rheumatoid and psoriatic arthritis, in particular, lead to chronic inflammation of the synovial membrane and its bone-associated ligaments and tendons, finally destroying the joints. Changes may be visible like knobby finger joints, but often the damage can only be visualized on X-ray [5].

The bony skeleton is a multifunctional organ characterized by calcified hard tissue composed of type I collagen and calcium phosphate deposited in a highly organized manner. An essential component of the skeletal system, it enables locomotive activity, stores calcium and harbors haematopoietic stem cells. Bones provide mechanical support to soft tissues, act as levers for muscles, protects internal organs along with the central nervous system, releases a variety of ions to maintain proper ionic balance in the body fluids and houses the bone marrow for producing blood cells. Bone remodeling through resorption and formation ensures proper functioning of the skeletal system. Osteoclasts, derived from the monocyte/ macrophage haematopoietic lineage are specialized cells that develop and adhere to bone matrix, secreting acid and lytic enzymes that degrade in it a specialized, extracellular compartment. They are essential for physiologic remodeling of bone and also play important physiologic and pathologic roles in the dentofacial complex. Osteoblasts synthesize the bone matrix and coordinated resorption of bone is carried out by osteoclasts, constituting morphogenesis and remodeling of bone. In normal physiological conditions, bone homeostasis is maintained by balancing the bone formation activity of osteoblasts and bone destruction by osteoclasts. The whole purpose of this is to redistribute bone tissue and remove excess calcium, thus maintaining the proper shape and structure of bone [1].

There is enough evidence to suggest that osteoclasts are implicated in the pathogenesis of rheumatoid arthritis [6]. Rheumatoid arthritis associated bone destruction is attributed principally to the abnormal activation of osteoclasts. Osteoclasts make and secrete digestive enzymes that break up or dissolve the bone tissue. They are found in areas of pannus invasion into bone at the sites of osteoclasia in both animal models and patients suffering from rheumatoid arthritis, playing an essential role in bone loss and distruction. Periarticular bone degradation is caused by osteoclasts. Implication of osteoclasts in inflammatory joint diseases have been reported as early as the 19th century, but the molecular characteristics of these cells in rheumatic patients was first described in details by Gravallese EM, et al. in the late 1990s [7]. Their findings suggested that osteoclasts express CD68, TRACP, cathepsin K, and the calcitonin receptor as multinucleated cells and appear at the interface of inflammatory synovial tissue and periosteal surface and eventually form resorption lacunae. Bone resorption is a result of osteoclast activity. Determinants of physiologic and pathologic roles of osteoclasts are not well understood to this day. Evidence suggests that osteoclasts are involved in the pathogenesis of focal bone erosion, a feature of rheumatoid arthritis. Patients with rheumatoid arthritis have a higher risk of fracture as a result of progressive bone deterioration leading to systemic osteoporosis in the development process. Bone loss is not only observed as erosion in the affected joints, but also in the forms periarticular and systemic 
osteoporoses. Importantly, inflammatory cytokines directly act on osteoclast precursor cells of hematopoietic lineage and activate their differentiation into osteoclasts by cooperating with RANKL, but it stills remains to be fully understood how these cytokines exert their direct effect. Although the higher circulation level of these cytokines may contribute to enhanced osteoclastogenesis in bone tissues apart from inflammatory lesions, the mechanism of systemic osteoporosis is associated with arthritis also remains unclear $[6,7]$.

Studies conducted on the immune regulation of osteoclasts in rheumatoid arthritis have given rise to the new research field of 'osteoimmunology', which attempts to investigate the interplay of the skeletal and immune systems at the molecular level. In making advancements in the field of treatment of diseases associated with bone and/or immune systems, this interdisciplinary field has proven its worth [8].

\section{REGULATION OF OSTEOCLASTS: FORMATION, DIFFEREN- TIATION AND PATHOLOGY}

Tehrani S, et al. described that every year nearly $10 \%$ of total human bone mass is reassembled. A fine balance between bone formation by osteoblasts and bone resorption by osteoclasts contribute to bone homeostasis. While osteoclasts arise from mesenchymal stem cells, they are known to differentiate from monocyte/macrocyte precursors of the hematopoietic system. A wide variety of hormonal changes and disturbances in growth factors can lead to skeletal abnormalities that can manifest as either decreased (osteoporosis) or increased (osteopetrosis) bone mass and comprise one of the hallmarks of imbalance between osteoblast and osteoclast activity [9]. Osteoblasts play a central role in regulating osteoclast maturation through receptor activator of nuclear factor-кВ ligand (RANKL) expression, as claimed by Tanaka Y, Bone degradation is caused through the secretion of cathepsin $\mathrm{K}$ and matrix metalloproteinase (MMP) like proteolytic enzymes by osteoclasts along with subsequent decalcification of the inorganic components of bone through the release of hydrochloric acid [10].

Boyce BF described osteoclasts as multinucleated cells arising from bone marrow through fusion of myeloid hematopoietic precursors. Chemokines in the bloodstream attract osteoclast precursor cells from the bone marrow into the systemic circulation, only to be attracted again at various resorption sites that release a variety of factors known as bone remodeling units (BRU). It is at these resorption sites they undergo differentiation into osteoclasts [11]. Greenfield EM, et al. reported an indirect link between the stimulation of osteoclast activity and an up-regulation of osteoclast differentiation factor [12].

In the bone marrow, osteoclasts are held by chemokines, an effect that can be modified by tumor necrosis factor (TNF), allowing osteoclast precursors to move into the circulation in events where inflammation is present, as described by Boyce BF. Red blood cells and platelets secrete bioactive molecules like sphingolipid, sphingosine-1-phosphate (S1P) that attracts osteoclast precursors towards the systemic circulation [11]. Kikuta, et al. have discussed that osteoclast precursors have the ability to express receptors for S1P having both chemo attractant and repellant properties. There is evidence to suggest that osteoclasts express S1P as well, allowing fusion with osteoclast precursors [13].

Kituka J, et al. also reported increased levels of S1P in the synovial fluid of patients with rheumatoid arthritis and concluded that this could be the reason why osteoclast precursors are attracted to joints affected by this condition. To prove their statement, they treated mice with inflammatory arthritis with a S1P receptor agonist and found that joint destruction had reduced significantly. This led to the hypothesis that the agonist helped retain osteoclast precursors and other immune cells in the bloodstream [13].

Osteoclast formation is also regulated by numerous cytokines and hormones, sources of which range from macrophages to osteoblasts and stromal cells. One of the first critical factors to be identified to play a pivotal role in the proliferation and differentiation of osteoclast progenitors is the macrophage colony stimulating factor (M-CSF), as reported by Duong LT and Rodan GA.Studies conducted on osteopetrotic mice suggest that since these species are unable to produce M-CSF, they lack osteoclasts completely, thus proving that M-CSF and the osteoblasts or stroma cells involved in their production are essential for the development of osteoclasts. In its early stages, M-CSF facilitates the expression of RANK from myeloid progenitors. M-CSF binds to its receptor to initiate a series of events that lead to the regulation of various other activities associated with osteoclasts and their precursors, as suggested by Boyce, et al. M-CSF has also been demonstrated to play a role in the regulation of activity of mature osteoclasts, since it has the ability to induce fusion of osteoclasts and affect their survival and chemotaxis in vitro. But according to Duong LT, et al. 
M-CSF does not itself alone control osteoclast maturation. This is where the role of osteoclast differentiation factor (RANKL) comes in [14].

Another mediator of osteoclast function is receptor activator of nuclear factor- $\mathrm{BB}$ (RANK) which is very similar to CD40 and is expressed on both osteoclast precursor cells and mature osteoclasts. Both RANK and RANKL facilitate the development and activation of osteoclasts and are deeply implicated in the process of bone loss. Jones $\mathrm{DH}$, et al. claim that inhibition of RANKL can effectively prevent the loss of bone tissue and cartilage at inflamed joints. RANKL can be expressed by a wide variety of cell types, including osteoblastic, chondroblastic and $\mathrm{T}$ and $\mathrm{B}$ cells, although sources that provide RANKL in common bone diseases still remain to be identified. Involvement of RANKL in the activation of mature osteoclasts and in osteoclastogenesis in the presence of $\mathrm{M}$-CSF has been proven using in vitro culture media in various experiments as reported by Jones DH, et al. RANKL can also degrade TNF-receptor activating factor 3 (TRAF3), which itself plays a role in the degradation of NF- $\kappa B$ inducing kinase that sets a limit in the formation of osteoclasts. Numerous studies suggest the importance of RANKL and its receptor RANK expressed on osteoclast precursors in the process of osteoclastogenesis. When RANKL binds to its receptor, RANK undergoes trimerization and adapter molecules like TNF receptor associated factor (TRAF) family of proteins are recruited. This further leads to activation of mitogen activated protein kinases along with NF- кB [15].

Osteoprotegerin (OPG) was discovered in 1997 independently by Simonet WS, et al. and Tsuda E, et al., which demonstrated an inhibitory activity on osteoclasts in vitro $[16,17]$. Today, osteoprotegerin is classified as a physiological regulator of bone resorption by osteoclasts. Functioning as a soluble decoy receptor to RANKL, it is known to inhibit the binding of RANKL to RANK, functioning as an effective inhibitor of osteoclast maturation and activation in vitro and in vevo. It is protein in nature and very similar to TNF family of receptors. Patients with bone disorders have demonstrated mutations in RANKL, RANK and osteoprotegerin [16, 17].

Amico LD, et al. studied the interaction between osteoclasts and T-cells and stated that activated T-cells secrete RANKL, which can be an important link between bone metabolism and the immune system since RANKL regulates osteoclastogenesis and remodeling of bone and several autoimmune diseases result in bone loss, including rheumatoid arthritis. Therefore, the infiltration of T-cells into the synovium can be regarded as a pathological hallmark of rheumatoid arthritis [18]. Kong, et al. reported that that T-cells express RANKL which directly acts on osteoclast precursor cells to induce osteoclastogenesis in vitro [19]. Focusing on the osteoclast modulatory activity of T-cells, Kiesel JR, et al. demonstrated that osteoclasts can provide T-cells with antigenic peptides leading to an inappropriate activation of an immune response. T-cells can also indirectly facilitate bone resorption through expression of pro-inflammatory cytokines that lead to expression of RANKL in non-T cells. Although RANKL inhibition cannot directly affect the inflammatory state, it can however, prevent bone loss and partially, cartilage [20]. RANKL expressed by T-cells bind to its receptor on the surfaces of osteoclasts, thus conveying a reverse signal in T-cells that can lead to increased apoptosis. In an area of inflammation, T-cells have the ability to differentiate into Th17 cells, which can induce osteoblasts to express RANKL, which provides a link between T-cell response and bone destruction in rheumatoid arthritis, as Amico LD, et al. describes. Th cells themselves also express RANKL and has a direct influence on osteoclastogenesis [18].

Synovial tissue samples from patients with rheumatoid arthritis have also been proven to be a source of various other factors that regulate bone resorption through osteoclast differentiation and activation, including tumor necrosis factor $\alpha$ (TNF- $\alpha$ ), interleukin 1 and 6 (IL-1 and IL6) that terminally lead to destruction of bone. A novel cytokine known as secreted osteoclastogenic factor of activated T-cells has been described by Rifas and Weitzmann, thought to induce osteoclast formation in the absence of RANKL and osteoblasts [21].

Okamoto K and Takayanagi H supports the theory that Th17 cells that produce IL-17 facilitate the expression of RANKL in synovial tissues, allowing osteoclasts to differentiate and become functional along with other inflammatory cytokines. So, to prevent bone damage by osteoclasts, inhibition of Th17 cells is deemed essential [22].

Okamoto $\mathrm{K}$ and Takayanagi $\mathrm{H}$, also demonstrated the formation of osteoclasts in synovial cell cultures obtained from rheumatic patients [22]. Gravallese EM, et al. detected the expression of RANKL in the synovium of such patients. Recent studies conducted on RANKL deficient mice provide 
genetic evidence to the fact that lack of osteoclasts protects these mice from bone destruction when arthritis is induced by serum transfer. This led Takayanagi to believe that antiRANKL and anti-osteoclast therapies could prove to be beneficial in treatment of bone damage in animal models of arthritis [7,22].

Gravallese EM, et al. was among the first to identify the molecular characteristics of osteoclasts in rheumatoid arthritis patients. He and his team demonstrated that multinucleated osteoclasts appear to settle at the interface of the inflamed synovial tissue and the surface of the periosteum to finally result in the formation of resorption lacunae. Macrophage lineage cells which are mononuclear, also migrate toward the bone surface. They concluded that osteoclasts abundantly populate in the chronically inflamed joints and are also produced locally by differentiation from their precursors. Accumulation of osteoclasts at the joints of other skeletal diseases further add to the fact that these cells are indeed, a common component of multiple forms of chronic inflammatory diseases [7].

According to Schett G, and Teitelbaum SL, the location of bone erosion is mainly governed by the anatomy of joints and is important in understanding the mechanism behind erosion. Lesions are likely to localize at the edges of bones where the synovial membrane fuses into the surface of the periosteum, an area that is close to the attachment sites of ligament and tendon sheaths and the proximal rim of articular cartilage. Bone erosions normally occur at radial sites of metacarpophalangeal joints that are juxtaposed to tendons, collateral ligaments and at sites of insertion of synovial membrane, which can be explained by these features. Sites where inflamed tendon sheaths pass bony joints are more likely the areas where osteoclasts and erosions initilally develop, as confirmed by animal models [23].

Proof that osteoclasts are responsible for structural damage in inflammatory arthritis can be found in the presence of osteoclasts within the resorptive lacunae. It has also been found through studies on ostepetrotic mice including those lacking RANKL or RANK that bone resorptive polykaryon also mediates degradation of bone and mineralized cartilage. Thus, to prevent joint destruction in rheumatoid arthritis patients it is necessary to directly or indirectly inhibit osteoclast formation and function. According to Goldring and Gravallese clinical trials currently focus on antiresorptive therapies and their effects on generalized bone loss. Agents like estrogen, calcitonin and biophosphates have proven to be effective in such trials [24].

Tanaka Shas stated that osteoclasts play an essential role in destruction of bone and cartilage, although the cellular mechanism is unclear [25]. Multinucleated cells at sites of pannus invasion into subchondrial bone were discovered by Gravallese EM, et al. that contained positive biomarkers for osteoclasts. Tartarate resistant acid phosphate (TRAP) is an important biomarker for osteoclasts and can be found among some multinucleated and mononucleated cells in the bone surface. Furthermore, giant multinucleated cells that are capable of differentiating into osteoclasts were isolated from the bone-pannus junctions of arthritic joints in collageninduced models of arthritis [7,25].

\section{OSTEOCLASTOGENESIS IN INFLAMMATORY ARTHRITIS}

An early event in the process of inflammatory joint disease is osteoclastogenesis, the development of osteoclasts from its precursors. Following induction of adjuvant or collagen induced arthritis, mononuclear precursors appear within 2 days while mature resorptive polykaryons take 5 days. A massive influx of monocyte/macrophage precursors of osteoclast into the affected joints marks a rapid and pronounced osteoclast formation. These cells subsequently undergo maturation through stimulation of expression of a variety of essential osteoclastogenic factors and turn into bone degrading cells inside the synovium. Abundance of macrophages in the joints of rheumatic patients further alleviates the condition. Influx of macrophages into the intraarticular space in controlled by chemokines, promoting their attachement to synovial micro vessels thus facilitating synovial membrane invasion. Chemokines such as CXCL-12, MIP-1 $\alpha$ and CCL-10 influence the mobilization of mononuclear cells in the bone marrow into the systemic circulation to be attracted eventually into the synovial membrane [26].

M-CSF and RANKL are required for the differentiation of osteoclasts from osteoclast precursors. RANK is present on the surface of the monocyte/macrocyte precursors. These two cytokines appear abundantly in the synovial membrane of rheumatoid arthritis patients, thus providing the optimum environment for osteoclastogenesis [26].

Increased RANKL has been reported in the synovium of rheumatoid arthritis patients and its inhibition by 
osteoprotegerin manipulation or by genetic processes can protect from bone erosions in animal models as described earlier. Kong YY, et al. states that similar results can be achieved through inhibition of M-CSF. Both RANKL and M-CSF are expressed by synovial fibroblast like cells, which can suggest their essential role in promoting bone destruction in rheumatoid arthritis. It is postulated by Kong YY, et al. that RANKL is also expressed by the activated T-lymphocytes in the rheumatic synovium which could be an important mechanism in the pathogenesis of inflammatory osteolysis. In light of current evidence, it is safe to say that in the context of inflammatory arthritis, a novel subset of CD4+ T-cells that produce IL-7 are the lymphocyte mediators of osteoclastogenesis [19]. Kotake S, et al. proposed that mesenchymal cells are stimulated by IL-7 to express RANKL. Two other factors that are abundant in rheumatic joints are TNF and IL-1, and thus play a major role in inflammatory osteolysis. In situations where there is an apparent abundance of TNF, such as in inflammatory joint disease, expression of RANKL and M-CSF is promoted in the mesenchymal cells to exert its osteoclastogenic effects and osteoclasts are recruited solely by directly targeting macrophage precursors, requiring a constitutive amount of RANKL . Induction of RANKL and M-CSF by TNF is essential to osteoclastogenesis, in situations where TNF is increased only moderately. In response to relatively small amounts of RANKL, precursors of osteoclasts are primed by TNF to undergo differentiation. This leads us to believe that TNF and RANKL are synergistically related in the process of osteoclastogenesis. Although scientists have proposed that TNF has the capacity to induce osteoclastogenesis independent of RANKL, Schett and Teitelbaum have suggested otherwise since RANK knockout mice fail to develop inflammatory osteolysis, which is opposed to the previous claim. TNF can also enhance the pool of macrophages which can stimulate osteoclast formation when mobilized to synovial joints. Surface molecules are expressed on macrophages through stimulation by proinflammatory cytokines to assist in osteoclastogenesis. TNF is also known to inhibit the differentiation and bone forming activity of osteoblasts in rheumatoid arthritis [27].

Expression of RANKL and RANK can also be induced by IL-1, just like TNF. Zwerina J, et al. has suggested that in the case of TNF driven synovial inflammation, mice deficient in IL-1 are protected from bone damage, which led them to believe that bone erosion is triggered through induction of IL-1 by TNF. Soluble osteoclastogenic factors that are produced abundantly in the inflamed joints are capable of moving into the systemic circulation, a phenomenon that could account for the glucocorticoid-independent osteoporosis in rheumatoid arthritis patients [28].

Although physiological bone resorption is followed by bone formation, this relationship is disrupted in inflammatory osteolysis, thus prohibiting the replacement of old skeletal tissue with new. Bone formation appears to be limited in rheumatoid arthritis while resorption is quite aggressive. Sclerosis from bone erosions may appear years after the onset of arthritis when inflammation is controlled therapeutically $[27,28]$.

Protective cytokines: Okamoto $\mathrm{K}$ and Takayanagi Hamong others, stated that a potent antagonist of osteoclast formation is interferon- $\gamma$ and cytokines of the TH1 lineage, including IL12 have the ability to suppress osteoclatogenesis. Formation of osteoclast can also be downregulated by IL-23. These cytokines, although activated in the synovium of rheumatic patients, are insufficient in achieving total control over bone resorption[29].

\section{STUDIES IN ANIMAL MODELS}

Although animal models are inherently limited, several rodent models have helped progress our understanding and have been used extensively to describe the fundamental mechanisms of rheumatoid arthritis and achieve some major advancements in treatment. Such models include the induced arthritis models, like collagen induced arthritis, collagen antibody induced arthritis among others and genetically manipulated models. These models have also allowed major advancements in understanding how multiple genetic and environmental factors influence the development of rheumatoid arthritis in susceptible individuals.

Gravallese EM described the blockade of osteoclasts in animal models of arthritis to prove that bone erosion is caused by osteoclasts and the critical role of RANKL in osteoclastogenesis, through the blockade of osteoprotegerin in the respective animal model. He observed that RANKL had been expressed in T-cells and in synovial cells of rats with adjuvant arthritis. Osteoprotegerin treatment at the onset of disease appeared to protect cortical and trabecular bone and cartilage in the treated rats as opposed to severe bone damage in untreated rats. The treatment was most effective when initiated at the early stages of pathogenesis [6]. 
Pettit AR, et al. conducted experiments on TRANCE/RANKL deficient mice and concluded that this strain is protected from bone erosion when arthritis is induced in them through serum transfer method. The activation of T-cells in this model of mice is bypassed and the animals are characteristically osteopetrotic. Although bone destruction was reduced, cartilage damage was present in both experimental and control groups. Their experiments confirm the pivotal role of osteoclasts in the pathophysiology of arthritis [30].

Zhao $\mathrm{H}$, et al. studied the effects of boldine isolated from Litsea cubeba on rats with collagen induced arthritis (CIA) through oral administration of boldine and observed the destruction of bone in the paws of these animals. They also cultured osteoclasts in vitro and subjected them to various levels of boldine concentration. They evaluated RANK expression through real time polymerase chain reaction. Boldine was found to have caused a decrease in ankle swellings and significantly prevented bone destruction to alleviate the pathological damage [31].

Another study conducted on carpal bone samples from post traumatic osteoarthritic race horses by Bertuglia A, et al. led to the conclusion that in carpal osteoarthritis, osteoclasts are recruited to subchondral bone with the possibility of inducing cartilage destruction. Cores harvested from the eqine third carpal bone were stained and scored and osteoclast density was calculated and compared to RANKL scores. A greater density of osteoclasts was reported in in the post traumatic osteoarthritic samples mainly in the subchondral bone plate. They concluded that during progression of spontaneous equine carpal post traumatic osteoarthritis, osteoclasts are recruited by cartilage RANKL, contributing to focal bone loss [32].

Zhang $\mathrm{H}$, et al. reported the contribution of myeloid-deprived suppressor cells in bone erosion using collagen induced arthritis models. These cells appear to be increased in collagen induced arthritis and later differentiate into osteoclasts. They also expand in the bone marrow of the collagen induced arthritis mice and contribute to bone destruction through stimulation by RANKL and M-CSF. Their results suggest that myeloid-deprived suppressor cells act as a source of osteoclast precursors and other inflammatory cytokines like IL-1 and contribute significantly to bone erosion in rheumatoid arthritis [33].

\section{CURRENT DEVELOPMENTS}

In light of recent developments it is safe to say that the upregulation of osteoclast differentiation factor or osteoprotegerin by osteoblasts is the primary mechanism that indirectly stimulates osteoclast activity, as reported by Greenfield EM, et al. [12]. RANKL and M-CSF can activate numerous signaling pathways, as confirmed by genetic experiments on mice and molecular studies. Boyce BF reports that osteoclasts and their precursors are also capable of regulating immune responses and osteoblast formation and function through direct cell interactions by means of ligands and receptors. Osteoclasts are currently gaining recognition as immune cells as they are likely to play a role in immune responses besides facilitating bone destruction [11]. Okamoto and Takayanagi have provided direct genetic evidence to the fact that RANKL deficient mice are protected from bone erosion when arthritis is induced in them through serum transfer [22]. Consistent with their findings, anti-RANKL and anti-osteoclast therapies have now been deemed beneficial in the treatment of bone damage in animal models of arthritis. The experiments of Zhao $\mathrm{H}$, et al. with boldine is also worth mentioning in this respect [31].

Recently in 2017, Hu F, et al. investigated the possibility of B10 cells to convert into cells that produce RANKL and impair their immunosuppressive functions to exacerbate the progression of rheumatoid arthritis. They observed an ectopic expression of RANKL by human regulatory B10 cells, which had a dramatic expansion under the influence of the rheumatic disease state. In the patients, these RANKL producing B10 cells were observed to promote osteoclast differentiation and bone erosion, which was not observed in healthy individuals, but these cells also declined in number with the increase of IL-10 producing regulatory B10 cells in patients undergoing remission. These researchers also suggested that TNF- $\alpha$ plays a central role in inducing B-10 cells to express RANKL and that the pathogenic conversion of these cells can be reverted following effective therapy [34].

In the past 30 years, there has been a tremendous growth in the number of therapeutic resources available for the treatment of rheumatoid arthritis. Currently included in the treatment strategy of rheumatoid arthritis are non-steroidal antiinflammatory drugs (NSAIDS), corticosteroids and disease modifying anti rheumatic drugs (DMARDs) of both synthetic and biological origins. The therapeutic goal is to alleviate the 
affected joints and prevent erosion, also providing clinical remission. Janus Kinase inhibitors are a novel drug class that have gained popularity in the treatment of arthritis [34].

\section{CONCLUDING REMARKS}

Although osteoclasts have been implicated as having a central role in bone destruction, the molecular mechanisms involved in the development of rheumatoid arthritis still remains unsolved and it is classified as a disease of unknown etiology. All the authors of their respective articles have identified osteoclasts as a major contributor to the inflammatory process, but they have not been able to provide sufficient evidence to the fact that other cell types might as well contribute to the process of osteoclastogenesis in rheumatoid arthritis. Galvellese EM has raised the question that enzymes produced by fibroblasts and macrophages in the synovium could in fact contribute to the process. The role of RANKL in osteoclastogenesis has been proven satisfactorily by various animal model studies as RANKL knockout mice failed to produce osteoclasts. But other cytokines as well may play a significant role in the process of osteoclastogenesis and the attention of scientists has been drawn towards TNF- $\alpha$ as an important driving force in the process. The dire consequences of TNF- $\alpha$ activated osteoclastic bone resorption remains to be explained. Furthermore, the role of the RANKL/RANK/ osteoprotegerin system in the destruction of cartilage still remains a question to be answered. There has been much skepticism on how osteoclast formation is turned off by interferon- $\gamma$, IL-12, IL-23 and osteoprotegerin in rheumatoid arthritis. There should be further clarification on these processes to develop new therapeutic interventions in the field of rheumatology [6].

Despite having a lot of unanswered questions, there has been a rapid advancement in the field of rheumatoid arthritis therapy with molecular target based medicine which has been possible through the numerous studies of osteoclasts and their precursors in the pathogenesis of arthritis. It has been proven by Redlich $\mathrm{K}$, et al. that bone resorption is completely prevented in TNF-mediated arthritis in the absence of osteoclasts, thereby proving that osteoclasts are indeed essential in bone destruction and their inhibition could stop the progression of rheumatoid arthritis. A lot has been achieved in understanding the mechanism of bone destruction in rheumatoid arthritis, and the molecular mechanism of the disease pathogenesis will hopefully be discovered in the near future[35]. At present, much research is being carried out on developing drugs that could selectively block osteoclast differentiation and function in the joints, as Schett and Teitelbaum states [23]. The research work of Zhao, $\mathrm{H}$ et al. has shown the immense potential of boldine (an isoquinoline alkaloid extracted from the roots of Litsea cubeba) in improving the micro-architecture of bonein arthritis. Although the effect of boldine in rheumatoid arthritis remains unclear, it is proven that boldine has a great potential in suppressing inflammatory responses and protecting bone, without any adverse effects on the liver or kidney [31]. According to the work of $\mathrm{Hu} \mathrm{F}$, et al. investigation into the immunosuppressive effects of B-10 cells could provide revolutionary insight into the treatment of autoimmune diseases such as rheumatoid arthritis. Biophosphonates have the potential of strongly inhibiting bone resorption and has proven to be a promising alternative in the treatment of rheumatoid arthritis. Inhibition of RANKL by desomumab is also promising. The emergence of cytokine mediated therapy represents one of the greatest advances in the field of autoimmune diseases including rheumatoid arthritis [34]. It is evident from the works of Ochi $S$, et al. and others that inflammation and bone destruction could be averted significantly with the use of anti-TNF- $\alpha$ antibodies [36].

Monocyte/macrophage lineages and hematopoietic stem cells are terminally differentiated into osteoclasts under the influence of M-CSF and RANKL. For bone resorption, maintenance of bone structure, function and remodeling, the differentiation of osteoclast precursors into mature osteoclasts is essential, since osteoclast exclusively mediates the bone remodeling procedure. However, excessive osteoclasts can be formed through exaggerated expansion of osteoclast precursors and can lead to bone erosion and arthritis. Specific antagonists of osteoclast differentiation and function may be discovered in the near future, which will further facilitate prevention of joint damage in rheumatoid arthritis.

\section{REFERENCES}

1. Kumar V, Abbas AK, Aster JC. (2015). Robbins and Cotran pathologic basis of disease (Ninth edition.). Philadelphia, PA: Elsevier/Saunders.

2. Senthelal S, Li J, Goyal A, et al. Arthritis. [Updated 2021 Aug 11]. In: StatPearls [Internet]. Treasure Island (FL): StatPearls Publishing; 2021 Jan-. Available from: https:// www.ncbi.nlm.nih.gov/books/NBK518992/ 
3. Arthritis and Rheumatic Diseases NIMH, October 2014.

4. Ahmed M, Haq SA, Islam MN, Banik SK, Alam MN. (2014). Burden of rheumatic diseases in a rural community of Bangladesh. Journal of Medicine. 15(2):125-130.

5. Ahmed S, Haq SA, Az-qadir AZ, Rahman MM, Paul S. (2017). SAT0713 Survey on prevalence of rheumatic disorders in Bangladeshi adults. Annal of Rheumatic Diseases. 76(2):1044-1045.

6. Gravallese EM. (2002). Bone Destruction in Arthritis. Ann Rheum Dis. 61(Suppl II):ii84-ii86.

7. Gravallese EM, Manning C, Tsay A, Naito A, Pan C, et al. (2000). Synovial tissue in rheumatoid arthritis is a source of osteoclast differentiation factor. Arthritis and Rheumatology. 2:250-258.

8. Christian G, Sascha G, Christof B, Wirtz DC, Schildberg FA. (2020). Osteoimmunology: A Current Update of the Interplay Between Bone and the Immune System. Frontiers in Immunology. 11:58.

9. Tehrani S, Faccio R, Chandrasekar I, Ross FP, Cooper JA. (2006). Cortactin Has an Essential and Specific Role in Osteoclast Actin Assembly Mol Biol Cell. 17(7):28822895.

10. Tanaka Y, Nakayamada S, Okada Y. (2005). Osteoblasts and osteoclasts in bone remodeling and inflammation. Curr Drug Targets Inflamm Allergy. 4(3):325-328.

11. Boyce BF. (2013). 'Advances in Regulation of Osteoclasts and Osteoclast Function.'J Dent Res. 92(10):860-867.

12. Greenfield EM, Bi Y, Miyauchi A. (1999). Regulation of Osteoclast activity Life Sci. 65(11):1087-1102.

13. Kikuta J, Iwai K, Saeki Y, Ishii M. (2011). S1P-targeted therapy for elderly rheumatoid arthritis patients with osteoporosis. Rheumatol Int. 31:967-969.

14. Duong LT, Rodan GA. (2001). Regulation of osteoclast formation and function. Reviews in Endocrine and Metabolic Disorders. 2:95-104.

15. Jones DH, Kong YY, Penninger JM. (2002). Role of RANKL and RANK in bone loss and arthritis.' Ann Rheum Dis; 61: 32-39.

16. Simonet WS, Lacey DL, Dunstan CR, Kelley M, Chang MS, et al. (1997). Osteoprotegerin: a novel secreted protein involved in the regulation of bone density. Cell. 89:309-319.
17. Tsuda E, Goto M, Mochizuki S, Yano K, Kobayashi F, et al. (1997). Isolation of a novel cytokine from human fibroblasts that specifically inhibits osteoclastogenesis. Biochem Biophys Res Commun. 234:137-142.

18. Amico LD, Roato I. (2012). Cross talk between T-cells and osteoclasts in bone resorption. Bonekey Rep. 1:82.

19. Kong YY, Feige U, Sarosi I, Bolon B, Tafuri A, Morony S, et al. (1999). Activated T cells regulate bone loss and joint destruction in adjuvant arthritis through osteoprotegerin ligand. Nature. 402:304-309.

20. Kiesel JR, Buchwald ZS, Aurora R. (2009). Crosspresentation by osteoclasts induces FoxP3 in CD8+ T cells. J Immunol. 182:5477-5487.

21. Rifas L, and Weitzmann MN. (2009). A novel T cell cytokine, secreted osteoclastogenic factor of activated $\mathrm{T}$ cells, induces osteoclast formation in a RANKL independent manner. Arthritis Rheum. 60(11):3324-3335.

22. Okamoto K, Takayanagi H. (2011). Osteoclasts in arthritis and Th17 cell development. International Immunopharmacology. 11:543-548.

23. Schett G, Teitelbaum SL. (2009). Osteoclasts and Arthritis Journal of Bone and Mineral Research. 24:1142-1146.

24. Goldring SR, Gravallese EM. (1999). Mechanisms of bone loss in inflammatory arthritis: diagnosis and therapeutic implications. Arthritis Research and Therapy. 2:33.

25. Tanaka S. (2013). Regulation of bone destruction in rheumatoid arthritis through RANKL-RANK pathways. World J Orthop. 4(1):1-6

26. Schett G. (2007). Cells of the Synovium in Rheumatoid Arthritis. Osteoclasts. Arthritis research and therapy. 9:203.

27. Kotake S, Sato K, Kim KJ, Takahashi N, Udagawa N, et al. (1996). Interleukin-6 and soluble interleukin-6 receptors in the synovial fluids from rheumatoid arthritis patients are responsible forosteoclast-like cell formation. J Bone Miner Res. 11(1):88-95.

28. Zwerina J, Redlich K, Polzer K, Joosten L, Kroenke G, et al. (2007). TNF-induced Structural Joint Damage Is Mediated By Interleukin-1. Proc Nat Acad Sci. 104:11742-11747.

29. Okamoto K, and Takayanagi H. (2011). Osteoclasts in arthritis and Th17 cell development. International Immunopharmacology. 11:543-548. 
30. Petit AR, Ji H, Stechow DV, Muller R, Goldring SR, et al. (2001). Transe/RANKL knockout mice are protected from bone erosion in a serum transfer model of arthritis. American Journal of Pathology. 159(5):1689-1699.

31. Zhao H, Xu H, Qiao S, Lu C, Wang G, et al. (2017). Boldine isolated from Litsea cubeba inhibits bone resorption by suppressing the osteoclast differentiation in collageninduced arthritis. International Immunopharmacology. 51:114-123.

32. Bertugila A, Lacourt M, Girard C, Beauchamp G, Richard H, et al. (2016). Osteoclasts are recruited to the subchondral bone in naturally occurring post-traumatic equine carpal osteoarthritis and may contribute to cartilage degradation. Osteoarthritis and Cartilage. 24:555-566.

33. Zhang H, Huang Y, Wang S, Fu R, Guo C, et al. (2015). Myeloid-deprived suppressor cells contribute to bone erosion in collagen induced arthritis by differentiating to osteoclasts. Journal of Autoimmunity. 65:1-8.
34. Hu F, Liu H, Liu X, Zhang X, Xu L, et al. (2017). Pathogenic conversion of regulatory B10 cells into osteoclast-priming cells in rheumatoid arthritis. Journal of Autoimmunity. 76:53-62.

35. Redlich K, Hayer S, Ricci R, David JP, Akrad MT, et al. (2002). Osteoclasts are essential for TNF- $\alpha$ mediated joint destruction. J Clin Invest. 110:1419-1427.

36. Ochi S, Sinohara M, Sato K, Gober SJ, Koga T, et al. (2007). Pathological Role of Osteoclast Costimulation in Arthritisinduced Bone Loss. PNAS. 104:11394-11399. 